\title{
Corrado Segre and the courses on higher geometry in Italy
}

\author{
Ciro Ciliberto · Claudio Fontanari
}

Published online: 25 January 2014

(c) Centro P.RI.ST.EM, Università Commerciale Luigi Bocconi 2014

\begin{abstract}
Corrado Segre drafted careful notes for a course of Higher Geometry that he taught in Torino in 1890-91, where he taught the theory of algebraic curves, later set out by him in a more comprehensive paper published in the Annali di Matematica Pura e Applicata. We put this in perspective, briefly comparing its contents to those of similar courses taught by Federigo Enriques in Bologna in 1897-98, Guido Castelnuovo in Rome in 1922, and to Francesco Severi's book Geometria delle serie lineari of 1926.
\end{abstract}

Keywords Corrado Segre - Guido Castelnuovo · Federigo Enriques · Francesco Severi - Algebraic geometry $\cdot$ Curves $\cdot$ Surfaces $\cdot$ Higher geometry

In Torino in the 1890-91 academic year Corrado Segre taught the course entitled 'Introduction to geometry of simply infinite algebraic entities'. In the memoir with almost the exact same title, published in the Annali di Matematica pura ed applicata [2], Segre said he was setting forth the theory of algebraic curves following the geometric method 'worked out for a course of lessons in geometry of the simply infinite algebraic entity taught in the year $1890-91^{\prime}$. In a note he added:

C. Ciliberto $(\square)$

Dipartimento di Matematica, Università di Roma Tor Vergata,

Via della Ricerca Scientifica, 00173 Rome, Italy

e-mail: cilibert@axp.mat.uniroma2.it

C. Fontanari

Dipartimento di Matematica, Università degli Studi di Trento,

Via Sommarive 14, 38123 Povo, TN, Italy

e-mail: fontanar@science.unitn.it
Those lessons, alongside the geometric method set forth here, also treated misprint the Riemannian method and that of Brill and Noether. In fact, the topic is such that it is not well treated unless it is developed under several aspects. The fact that I have taken it upon myself here to expound it from the geometric point of view is not to be interpreted in the sense of a preference that in my opinion must be given to this method with respect to the others. All of them deserve to be studied; each has its own special merits; for each there are questions in which they go further, or at least manage to be more illuminating than the others. But the geometric method to which I permit myself to call attention is precisely the one that is less well known up to now.

An analogous tripartite treatment is also the basis of the treatise by Franceso Severi, Geometria delle serie lineari [4], which ambitiously proposes itself as a systematic exposition of "everything that is important in the field of algebraic geometry'. On page VII, we read:

It is my desire that this Treatise be methodical and that each question be developed in a way that is exhaustive and rigorous, in part because it is necessary to discredit the legend that in algebraic geometry the lack of rigour and determinancy is almost a necessity.

Here, however, the geometric method (which Severi defines 'the hyperspace thread, due to Segre and Castelnuovo', presented in the seventh chapter) and the 'more strictly algebraic' method of Brill and Noether (to which is dedicated the eighth chapter), instead of appearing with the 'Riemannian method' of a complex analytic nature, is flanked by a new algebraic-geometric method "which we 
have called "rapid method" because it arrives more rapidly to the central properties of the theory and is indicated by the Author' [3].

The courses in higher geometry taught in the meantime in Italian universities are situated along this same line of thought, even while introducing into the program significant variants, expressions of the professors' personalities. In the course taught in Bologna in the 1897-98 academic year by Federigo Enriques (described in [1]), after two chapters traditionally dedicated to plane algebraic curves and to geometry on a curve up to the Riemann-Roch theorem, and to the classification of curves of the first species "curves of the first genera" ("with the tendencies to make more evident the algebraic content of the definitions and the theorems. Experience has shown me that this also contributes to clarity; thus, for example, it seems to me that the algebraic definition of the erase "the" linear series, by means of the erase "the" rational functions on a curve, turns out to be more illuminating than any other'), then dedicates a third chapter to the geometry of rational algebraic surfaces: from 'rational or Cremonian transformations between two planes' to the 'theorem of Noether on the surfaces containing a linear sheaf of rational curves'.

Guido Castelnuovo, instead, in the last course he taught in Rome on higher geometry, entitled 'Plane and skew algebraic curves' (in the 1922-23 academic year, before he transferred direi "before moving" to the chair of complementary mathematics), follows the usual first three parts (entitled respectively 'Plane algebraic curves', 'Algebraic correspondences' and 'Geometry on curves', with a fourth part on 'Skew curves', which arrives to the treatment of the 'maximum species "genus" invece di "species" of a skew curve $C_{n}$ ' (that is, contained in an $n$-dimensional hyperspace) 'without multiple points' (the celebrated 'Castelnuovo's Bound') and the 'number of parameters on which skew curves depend'.

Translated from the Italian by Kim Williams.

\section{References}

1. Enriques, F.: Programma di un corso di geometria superiore per l'anno 1897-98. Bollettino di bibliografia e storia delle Scienze matematiche II, 76-78 (1899)

2. Segre, C.: Introduzione alla geometria sopra un ente algebrico semplicemente infinito. Annali di Matematica pura ed applicata, s. II, XXII, 41-142 (1894)

3. Severi, F.: Sulle intersezioni delle varietà algebriche e sopra i loro caratteri e... algebrica. Atti Reale Istituto Veneto di Scienze e Lettere 79, 929-938 (1920)

4. Severi, F.: Geometria delle serie lineari. Zanichelli, Bologna (1926)

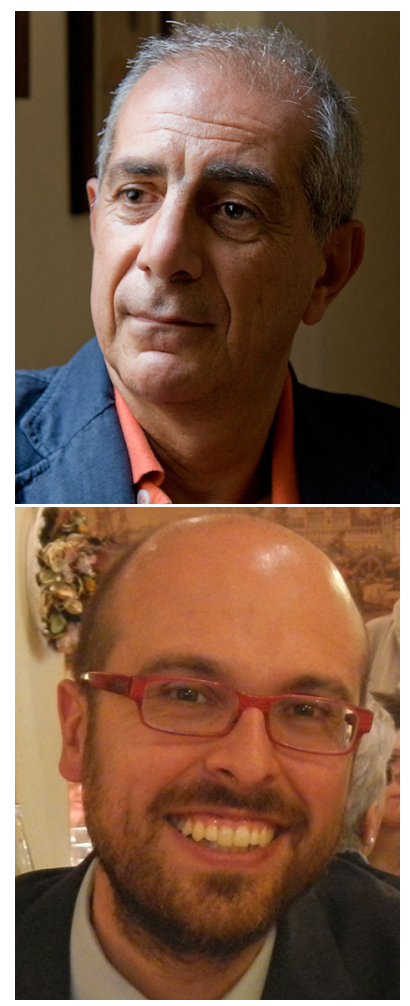

Ciro Ciliberto teaches geometry at the Università di Roma Tor Vergata. His main scientific interests are in algebraic geometry.

Claudio Fontanari is a full professor per quel che ne so Fontanari e' "associate professor" of geometry at the University of Trento. He is adjunct secretary of the ècancellare è Unione Matematica Italiana. His research concerns algebraic geometry, and in particular the study of curves and their moduli spaces. 